\title{
Haploid, diploid, and pooled exome capture recapitulate features of biology and paralogy in two non-model tree species
}

$9 \quad$ 33iodiversity Research Centre, University of British Columbia, Vancouver, BC, Canada

$10 *$ corresponding author

$11 \quad$ \# equal contribution

12 Running Title: Validating pool-seq data in conifers

13 Keywords: pool-seq, non-model, paralogy, Pinaceae, exome-capture

\section{Corresponding Author}

15 Brandon Lind

162424 Main Mall

173027 Forest Science Centre

18 University of British Columbia

19 Vancouver, BC, Canada

20 Email: lind.brandon.m@gmail.com

October 6, 2020

${ }^{1}$ Centre for Forest Conservation Genetics and Department of Forest and Conservation

${ }^{2}$ Department of Biological Sciences, University of Calgary, Calgary, AB, Canada 


\section{Abstract}

22 Despite their suitability for studying evolution, many conifer species have large and 23 repetitive giga-genomes $(16-31 \mathrm{Gbp})$ that create hurdles to producing high coverage SNP

24 datasets that captures diversity from across the entirety of the genome. Due in part to 25 multiple ancient whole genome duplication events, gene family expansion and subsequent

26 evolution within Pinaceae, false diversity from the misalignment of paralog copies create

27 further challenges in accurately and reproducibly inferring evolutionary history from

28 sequence data. Here, we leverage the cost-saving benefits of pool-seq and exome-capture

29 to discover SNPs in two conifer species, Douglas-fir (Pseudotsuga menziesii var. menziesii

30 (Mirb.) Franco, Pinaceae) and jack pine (Pinus banksiana Lamb., Pinaceae). We show,

31 using minimal baseline filtering, that allele frequencies estimated from pooled individuals

32 show a strong positive correlation with those estimated by sequencing the same population

33 as individuals $(\mathrm{r}>0.948$ ), on par with such comparisons made in model organisms.

34 Further, we highlight the use of haploid megagametophyte tissue in identifying sites that

35 are likely due to misaligned paralogs. Together with additional minor filtering, we show

36 that it is possible to remove many of the loci with large frequency estimate discrepancies

37 between individual and pooled sequencing approaches, improving the correlation further

38 ( $\mathrm{r}>0.973)$. Our work addresses bioinformatic challenges in non-model organisms with

39 large and complex genomes, highlights the use of megagametophyte tissue for the

40 identification of paralog sites when sequencing large numbers of populations, and suggests

41 the combination of pool-seq and exome capture to be robust for further evolutionary

42 hypothesis testing in these systems. 
Lind et al.

Validating pool-seq data in conifers

43

44

45

46

47

\section{1 | Introduction}

Quantifying the spatial structure of neutral and adaptive genetic variation within

ecologically and economically important tree species (and close relatives) is fundamental

to forecasting and managing their response to changing selection pressures from pests,

pathogens, and climate (Aitken et al. 2008; Alberto et al. 2013; Holliday et al. 2017; Janes

et al. 2017; Sniezko et al. 2017). Prerequisite to this information is the ability to produce

high quality and cost-effective data from which to generate reliable inference. While the

life history of many tree species offers some ideal circumstances for studying adaptive

evolution at the genetic level (Neale \& Savolainen 2004; Neale \& Kremer 2011), two

ancient whole genome duplication events in progenitors of Pinaceae lineages (Li et al.

2015), transposable element dynamics (Morse et al. 2009; Voronova et al. 2017), tandemly

arrayed genes (Pavy et al. 2017), subsequent gene duplication (Krutovsky et al. 2004,

Casola \& Koralewski 2018) and gene family expansion (e.g., Liu et al. 2016) have led to

giga-genomes $(>16 \mathrm{~Gb}$ in size) recalcitrant to chromosome-level genome assembly under

modern budget and computational constraints (Neale et al. 2017a). For example, analysis

of Pinus taeda L. (Pinaceae) has yielded estimates that upwards of $82 \%$ of its $22 \mathrm{~Gb}$

genome is repetitive (Wegrzyn et al. 2014). It is also thought to be rich in pseudogenes

(Kovach et al. 2010).

1 Such large genome sizes have hampered production of dense SNP datasets across a

2 large number of individuals (Lind et al. 2018). Most recent sequencing efforts in conifers 
Lind et al.

Validating pool-seq data in conifers

63 have either used some form of reduced representation sequencing such as restriction-site

64 associated DNA sequencing (i.e., RADseq; reviewed in Andrew et al. 2016 and Parchman

65 et al. 2018), which relies upon relatively few genomic resources, or targeted capture (e.g.,

66 Lu et al. 2016; Suren et al. 2016), which requires significant genomic and budgetary

67 resources including the design of capture arrays (but see Puritz and Lotterhos 2018). To

68 capture population-level polymorphism information while minimizing cost, the sequencing

69 of pooled individuals (i.e., pool-seq approaches) has emerged as a cost-effective alternative

70 to sequencing individuals (Gautier et al. 2013; Schötterer et al. 2014). Further, pool-seq

71 can be combined with targeted capture approaches to both reduce cost and sample specific

72 areas of the genome that are a priori considered functionally relevant (e.g., Rellstab et al.

$732019)$.

74 Pool-seq approaches use read counts across pooled individuals to estimate allele

75 frequencies (generally for a single population). A number of studies have empirically

76 evaluated the congruence between individual and pool-seq allele frequency estimates

77 across various taxa (e.g., Futschik \& Schlötterer 2010; Rellstab et al. 2013, 2019;

78 Fracassetti et al. 2015). Such studies have led to broad agreement on the accuracy of pool-

79 seq, with best practices following Schlötterer et al. (2014) Of exceptional significance for

80 the estimation of allele frequency from read count data is the proper alignment of reads

81 to the reference. Misalignments, which may be particularly important for exome capture

82 data, can be due to reads from paralogs in the data mapping to the incorrect copy in the 
Lind et al.

Validating pool-seq data in conifers

83 reference, or from paralog copies being collapsed into a single sequence in the reference

84 assembly where copies in the data map to this single sequence. These misalignments will

85 skew allele ratios and bias allele frequency estimates downstream. In particular for non-

86 model species with histories of whole genome duplication or gene family expansion, steps

87 must be taken to categorize misalignments so that there are not substantial allele

88 frequency biases in downstream datasets.

89 Here we harness the multicellular haploid megagametophyte of conifers to aid in

90 mapping and analyzing poolseq data from diploid individuals. We use this pooled exome

91 capture approach for two conifers: coastal Douglas-fir (Pseudotsuga menziesii var.

92 menziesii (Mirb.) Franco, Pinaceae) and jack pine (Pinus banksiana Lamb., Pinaceae), to

93 evaluate the utility of pool-seq approaches in these systems. We use sequence data from

94 individuals to validate allele frequency estimates of the same individuals in pools, and use

95 haploid sequencing to identify misalignments from paralogous sites to quantify their effects

96 on the congruence between individual and pool-seq estimates (Table 1). Together, these

97 datasets provide a path forward for filtering pool-seq data of this kind, particularly for

98 studies of non-model organisms using a diverged, and potentially fragmented, reference

99 genome. Our methods further highlight a cost-effective means to empirically isolate

100 potentially misaligned paralogs in species with accessible haploid tissue. 
TABLE 1 Description of datasets used to call SNPs for both Douglas-fir and jack pine. indSeq ${ }^{\dagger}$ and poolSeq datasets for a given species share the same individuals from a single population. The megaSeq dataset consists of haploid megagametophyte tissue from a single individual not included in the indSeq or poolseq datasets.

\begin{tabular}{lccc}
\hline Dataset & $\begin{array}{c}\text { Ploidy per sample } \\
\text { (number of } \\
\text { samples) }\end{array}$ & $\begin{array}{c}\text { SNP } \\
\text { Caller }\end{array}$ & Purpose \\
\hline indSeq & $2(20)$ & GATK4 & $\begin{array}{c}\text { Validate poolSeq allele frequency estimates; } \\
\text { calculate read ratio statistics to validate candidate } \\
\text { paralog misalignments }\end{array}$ \\
\hline poolSeq & $2(20)$ & VarScan & $\begin{array}{c}\text { Compare with indSeq SNP set to } \\
\text { determine filtering strategy }\end{array}$ \\
megaSeq & $1(1)$ & VarScan fon folse & $\begin{array}{c}\text { Identify heterozygous sites as candidates for false } \\
\text { SNPs due to misalignment of diverged / duplicated } \\
\text { paralogs }\end{array}$ \\
\hline
\end{tabular}

${ }^{\dagger}$ Note that we use camelCase to denote our datasets, and reserve hyphens (e.g., pool-seq) to denote methodologies.

\section{1 / Focal Species and Population Sampling}


Lind et al.

Validating pool-seq data in conifers

111 For both Douglas-fir and jack pine, we sampled 20 individuals for use in individual

112 and pooled sequencing sets from operational reforestation seedlots created from bulk open-

113 pollinated seeds from tens or hundreds of seed parents from a single provenance (see

114 Supplemental Note 1.1). We used a single jack pine seed to extract megagametophyte

115 haploid tissue. For Douglas-fir haploid data, we randomly chose paired-end fastq files from

116 a previously sequenced Douglas-fir megagametophyte taken from a single individual

117 (NCBI SRA accession SAMN0333061, Neale et al. 2017b) to match sequencing effort for

118 jack pine haploid tissue (Supplemental Note 1.2).

119 2.2 / Exome Capture Probe Design

120 The capture probes were designed based on the genes identified using RNA

121 sequencing (RNA-seq) data for Douglas-fir and jack pine. De novo transcriptome assembly

122 was performed for each species using RNA-seq reads. For jack pine, RNA-seq reads were

123 sequenced from a sample of needles from a single tree. For Douglas-fir, RNA-seq reads

124 were obtained from two sources: one source was the read sets deposited in NCBI SRA,

125 including SRX1851630 (Little et al. 2016), SRX1286745 (Hess et al. 2016), SRX1341335

126 (Cronn et al. 2017a), and SRX136240 (Cronn et al. 2017b). The other source was the

127 reads sequenced from two needle samples infected by the fungal pathogen Phaeocryptopus

128 gaeumannii, which causes Swiss needle cast disease in Douglas-fir trees. 
Lind et al.

Validating pool-seq data in conifers

RNA-seq was conducted by McGill University and Génome Québec Innovation

130 Centre using HiSeq2500 V4 in paired-end 125bp format. The raw reads were processed by

131 the software FASTX-Toolkit (v0.0.13, http://hannonlab.cshl.edu/fastx_toolkit),

132 including clipping the adaptors (-1 25), filtering the artifacts, and keeping the reads with

133 a minimum quality score of 20. The filtered reads were used to perform de novo

134 transcriptome assembly using the software Trinity v2.4.0 (--bowtie2, Grabherr et

135 al. 2011). Among the assembled transcripts, only the longest isoforms with a length of at

136 least $300 \mathrm{bp}$ for each gene were retained, which were then used as reference to align the

137 reads using the software RSEM (v1.3.0 Li and Dewey 2011). From the expression

138 quantification of transcripts, transcripts with aligned reads and transcript per million

$139(\mathrm{TPM}) \geqslant 1$ were retained. The completeness of the filtered transcripts was examined using

140 the 1,375 orthologs in the BUSCO (v3.0, Benchmarking Universal Single Copy Orthologs)

141 set of embryophyta_odb10 (--evalue 1e-10, Simão et al. 2015).

142 To avoid probes spanning exon-intron boundaries, exons were targeted to design

143 probes. Using the software GMAP (v2017-06-20, Wu and Watanabe 2005), the filtered

144 transcripts from Douglas-fir were aligned to the convarietal reference (Pseudotsuga

145 menziesii var. menziesii (coastal Douglas-fir; v1.0, Neale et al. 2017b). The jack pine

146 transcripts were aligned to the congeneric loblolly pine (Pinus taeda) reference genome

147 (v.1.01, Wegrzyn et al. 2014) as there is no available jack pine reference genome, and both

148 loblolly and jack pine belong to Pinus subgenus Pinus, the hard pines. Exon sequences 
Lind et al.

Validating pool-seq data in conifers

149 with a length of at least 100bp were submitted to Roche NimbleGen for Custom SeqCap

150 EZ probe design.

151

To evaluate the capture efficiency of the probes, the captured sequences were

152 aligned to reference genomes and numbers of reads on-target, near-target ( $\leqslant 500 \mathrm{bp}$ from

153 target regions), and off-target regions were counted using "intersect" function in the

154 software BEDtools v2.28.0 (-f 0.75, Quinlan and Hall 2010). The depth of captured

155 sequences was counted using "depth" function in the software SAMtools v1.3 (-q 30

$156-\mathrm{Q}$ 20, Li et al. 2009). The cumulative depth was calculated and plotted using R (R Core

157 Team 2018).

158 2.3 / DNA Extraction, Library Preparation, and Sequencing

159 In total, three datasets were created for each of the two species (Table 1) - note that

160 we use camelCase (e.g., poolSeq) to denote our datasets, and reserve hyphens (e.g., pool-

161 seq) to denote methodologies. These datasets included individual sequencing of 20 diploid

162 individuals from a single population (hereafter indSeq), the same individuals pooled

163 together prior to sequencing (hereafter poolSeq), and haploid megagametophyte tissue

164 sequenced from a single individual (hereafter megaSeq). We use the indSeq dataset to

165 validate allele frequency estimates from our poolSeq data, and the megaSeq data to probe

166 our data for heterozygote SNPs (i.e., potential false-positive SNPs) caused by the 
Lind et al.

Validating pool-seq data in conifers

167 misalignment of diverged paralogs that could affect our allele frequency estimates (Table

168 1; see also $\$ 2.6$ / Comparison of Sequencing Approaches).

169 For each dataset we extracted DNA from either diploid needle tissue or haploid

170 megagametophyte tissue (see Supplemental Note 1.3). From these extractions,

171 approximately 100ng of DNA from each individual or pooled DNA sample was used for a

172 barcoded (Kapa, Dual-Indexed Adapter Kit) library with an approximately 350-bp mean

173 insert size. SeqCap library preparation was performed using custom Nimblegen SeqCap

174 probes (described above in $\$ 2.1$ ) according to the NimbleGen SeqCap EZ HyperCap

175 Workflow User's Guide Ver 2 (Roche Sequencing Solutions, Inc., CA USA). Following

176 capture, each library was sequenced in a $150 \mathrm{bp}$ paired-end format on an Illumina

177 HiSeq4000 instrument at the Genome Quebec Innovation Centre at McGill University,

178 Montreal, Canada.

1792.4 / Bioinformatic SNP Calling Pipelines

180 Raw paired-end sequence reads from all datasets were trimmed with fastp (v0.19.5,

181 Chen et al. 2018) by trimming reads that did not pass quality filters of less than twenty

182 N's, a minimum mean Phred quality score of 30 for sliding windows of five base pairs, and

183 a final length of 75 base pairs with no more than 20 base pairs called as N (-n 20 -M 30

$184-\mathrm{W} 5-175-\mathrm{g}-3$ ). Trimmed reads were mapped with bwa-mem (v0.7.17, Li \& Durbin

1852009 ) to reference assemblies; we mapped jack pine to the loblolly reference (v2.01, 
Lind et al.

Validating pool-seq data in conifers

186 Wegrzyn et al. 2014) and Douglas-fir to the convarietal reference (v1.0, Neale at al.

187 2017b). The resulting . sam files were converted to binary with SAMtools v1.9 (view,

188 sort, index; Li et al. 2009) and subsequently filtered for proper pairs and a mapping

189 quality score of 20 or greater (view -q 20 -f 0x0002 -F 0x0004). Using Picardtools

190 v2.18.9 (http://picard.sourceforge.net), read groups were added and duplicates

191 subsequently removed from filtered bam files.

192 We then called SNPs using the Genome Analysis Toolkit (GATK v4.1.0.0; McKenna et

193 al. 2010) for indSeq data, and VarScan (v2.4.3; Koboldt et al. 2012) for both poolSeq

194 and megaSeq datasets (Table 1) for comparisons since future datasets that stem from a

195 larger project will all be poolSeq (and we will therefor only be using VarScan). For SNPs

196 called with GATK4, we used HaplotypeCaller (--genotyping-mode DISCOVERY -ERC

197 GVCF) and GenotypeGVCFs. We then filtered data with SelectVariants (--select-

198 type-to-include SNP), VariantFiltration (--filter-expression “QD $<2.0||$ FS

$199>60.0||$ MQ $<40||$ MQRankSum <-12.5"), and finally with vcftools v0.1.14 (--

200 maf 0.00 -mingQ 20 -max-missing 0.75; Danecek et al. 2011). BQSR was not carried

201 out in our analysis due to the lack of a high-quality reference set of SNPs for our species.

202 Note that no further filtering (e.g., for depth) was done for this initial baseline filtering

203 strategy (further filtering is described in $\S 3.4$ ).

204 Before calling SNPs with VarScan, we first realigned indels with GATK 3.8 (McKenna

205 et al. 2010) - RealignerTargetCreator then IndelRealigner - and then passed a 
Lind et al.

Validating pool-seq data in conifers

SAMtools mpileup object directly to VarScan::mpileup2cns with a minimum coverage

207 set to $8, p$-value $<0.05$, minimum variant frequency of 0.00 , ignoring variants with $>90 \%$

208 support on one strand, a minimum average genotype quality of 20 , and a minimum allele

209 frequency of 0.80 to call a site homozygous (--min-coverage 8 --p-value $0.05--$ min-

210 var-freq 0.00 --strand-filter 1 --min-avg-qual 20 --min-freq-for-hom 0.80$)$.

211 Output was then filtered with a custom python (v3.7, www.python.org) script to filter

212 out indels, keep only biallelic loci, and to ensure a genotype quality score $>20$. From the

213 megaSeq data, we then isolated heterozygous SNP calls (hereafter megaSNPs) that

214 represent errors in genotype calling given the haploid nature of the tissue sequenced - to

215 keep only heterozygous calls, we ignored any biallelic cases where only the non-reference

216 allele was called. Such apparent SNPs are likely false due to misalignments. We have

217 published our complete SNP calling pipelines in publicly available repositories

218 (https://GitHub.com/CoAdapTree/gatk_pipeline and./varscan_pipeline).

2192.5 / Validation of megaSNPS as indicators of paralogy artifacts

220 To check whether heterozygous sites (megaSNPs) called from VarScan megaSeq are

221 following expectations of patterns from paralogs, we investigated read ratio deviations

222 from a binomial expectation for these VarScan megaSNP sites at the same sites in our

223 GATK indSeq data using heterozygous diploid individuals (sensu McKinney et al 2017; see

224 also Rellstab et al. 2019). For true positive SNPs, heterozygous diploid individuals should 
Lind et al.

Validating pool-seq data in conifers

225 have, on average, an even ratio of reference (REF) and alternative (ALT) read counts. If

226 the SNP is due to a bioinformatic error arising from the misalignment of paralogs (i.e., a

227 false positive SNP), the read ratio will differ significantly from this expectation when there

228 is a SNP at a given position in only one paralog copy (McKinney et al. 2017). Similarly,

229 if there is a fixed difference at a given position between two copies, then all individuals in

230 a population will present as heterozygotes with balanced read counts for REF and ALT

231 at that site. If we are sequencing (and then post-hoc correctly identifying) paralogs in our

232 poolSeq data using megaSNP sites, misalignment of either duplicated or diverged paralogs

233 will cause read ratio deviations in these loci (and affect allele frequency estimates from

234 poolSeq, and downstream analyses), which we should be able to detect in our indSeq data.

235 As described by McKinney et al. (2017), subsequent to whole-genome duplication during

236 the rediploidization phase as homeologous chromosomes diverge, tetrasomically inherited

237 sets of paralogs (duplicates) organize into distinct disomic loci (diverged duplicates).

238 We calculated these read ratio statistics for sites within the intersection of (1)

239 megaSNPs, indSeq, and poolSeq SNPs, and (2) poolSeq and indSeq SNPs alone; hereafter

240 intersections I1 and I2. The purpose of (1) is to see how paralogs could affect our poolSeq

241 data (leveraging information in our indSeq data to do so), and of (2) is to visualize the

242 potential influence of paralogs in our data independent of sites identified as megaSNP

243 sites, as well as to compare poolSeq allele frequency estimates with those estimated from

244 the indSeq dataset. For these sites, we queried the indSeq data to record the frequency of 
Lind et al.

Validating pool-seq data in conifers

245 heterozygous individuals $(H)$, the allele depth ratio $\left(D=\frac{R E F \text { depth }}{\text { total depth of coverage }}\right)$, and the

246 deviation of allele depth from expectation (REF depth $-0.5^{*}$ total depth) standardized by

247 properties of a binomial distribution with $n=$ depth of coverage, and $p=0.5$ (i.e., the $z$ -

248 score for the allele ratio deviation) following McKinney et al. (2016, 2017). We compare

249 our results with simulations carried out by McKinney et al. 2017. We used custom python

250 code to replicate the methods of McKinney et al. (2016), which is available online at

251 https://github.com/CoAdapTree/testdata_validation/003 testdata_validate_megaSN

252 Ps.ipynb.

2532.6 / Comparison of sequencing approaches

254 To study the utility of our pooled exome capture approach, we compared estimates of

255 allele frequency from our indSeq data with estimates from our poolSeq data. To do so, we

256 took the baseline-filtered SNPs from poolSeq and indSeq (see $\$ 2.4 /$ Bioinformatic SNP

257 Calling Pipelines) and identified common SNPs (i.e., intersection I2). To quantify and

258 visualize congruence between allele frequencies estimated with these methods, we report

259 Pearson's correlation coefficient, plot histograms to visualize the congruence across the

260 minor allele frequency (MAF) spectrum, and further plot 2D histograms to visualize

261 congruence of allele frequency estimates. To visualize how filtering poolSeq SNPs affects

262 the congruence between indSeq and poolSeq allele frequency estimates, we plot the allele

263 frequency differences between methods (hereafter AFdiff, calculated as the difference in 
264 allele frequency methods of poolSeq and indSeq: $\left.p o o l S e q_{A F}-i n d S e q_{A F}\right)$ against poolSeq

265 MAF, poolSeq depth of coverage, $H$, and the $Z$-score of read ratio deviation.

\section{3 | Results}

\section{1 / Sequencing, Mapping, and Probe efficiency}

272 for indSeq, 130 million reads for poolSeq, 202 million reads for megaSeq). Mapping rates

273 generally reflected the phylogenetic relationship between the sequenced individuals and

274 the reference used, where rates were high for all coastal Douglas-fir datasets mapping to

275 the convarietal reference (mean 85.11\%) and relatively lower rates for jack pine datasets mapping to the congeneric Pinus taeda reference (mean 35.36\%; Table S1). 

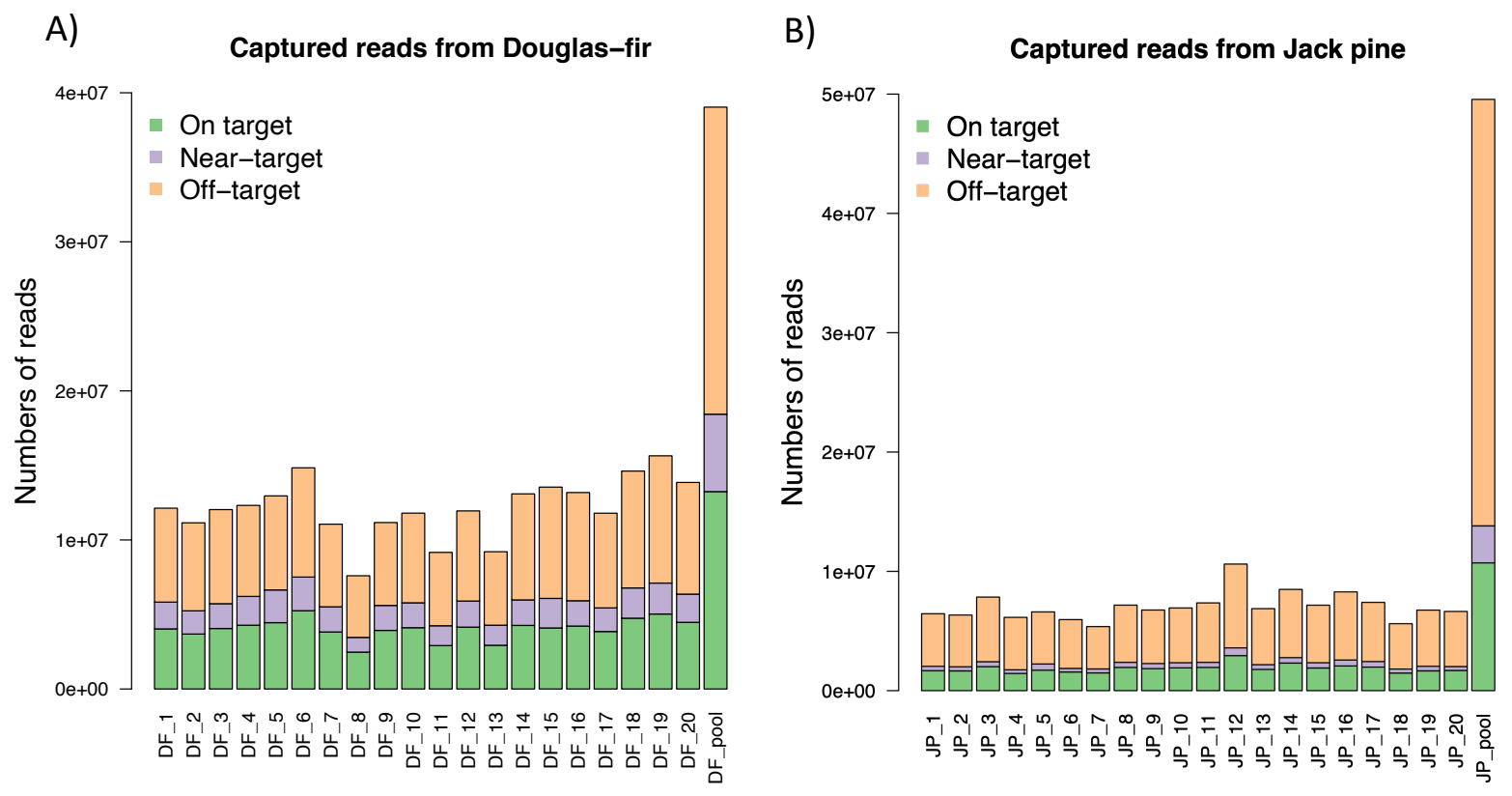

FIGURE 1 Numbers of captured reads from Douglas-fir (A) and jack pine (B) that mapped on target, near-target ( $\leq 500 \mathrm{bp}$ from target) and off-target regions. On the x-axis, from left to right, the first 20 bars represent indSeq samples, and the last bars represents the poolSeq samples.

in Douglas-fir. 
bioRxiv preprint doi: https://doi.org/10.1101/2020.10.07.329961; this version posted October 8, 2020. The copyright holder for this preprint (which was not certified by peer review) is the author/funder. All rights reserved. No reuse allowed without permission.

Lind et al.

Validating pool-seq data in conifers

A)

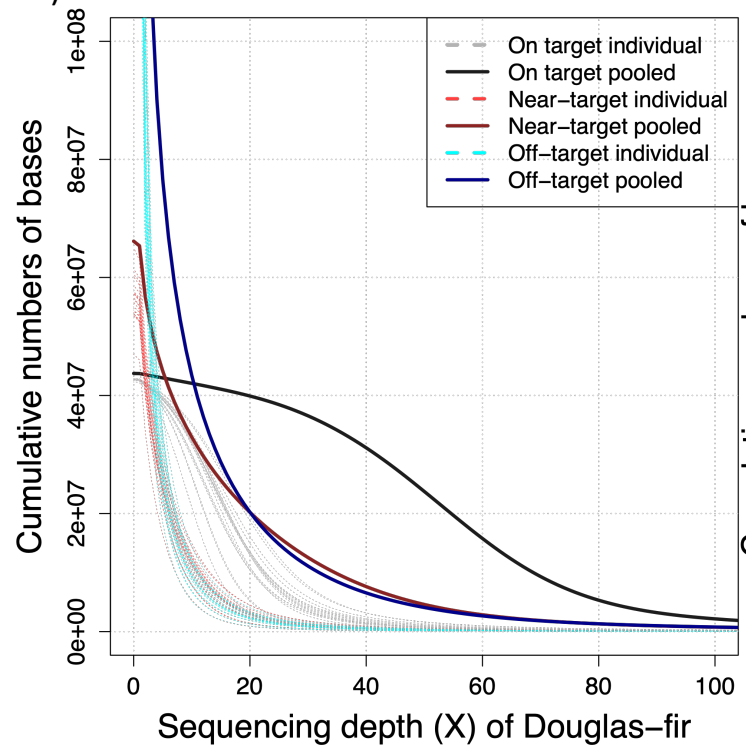

B)

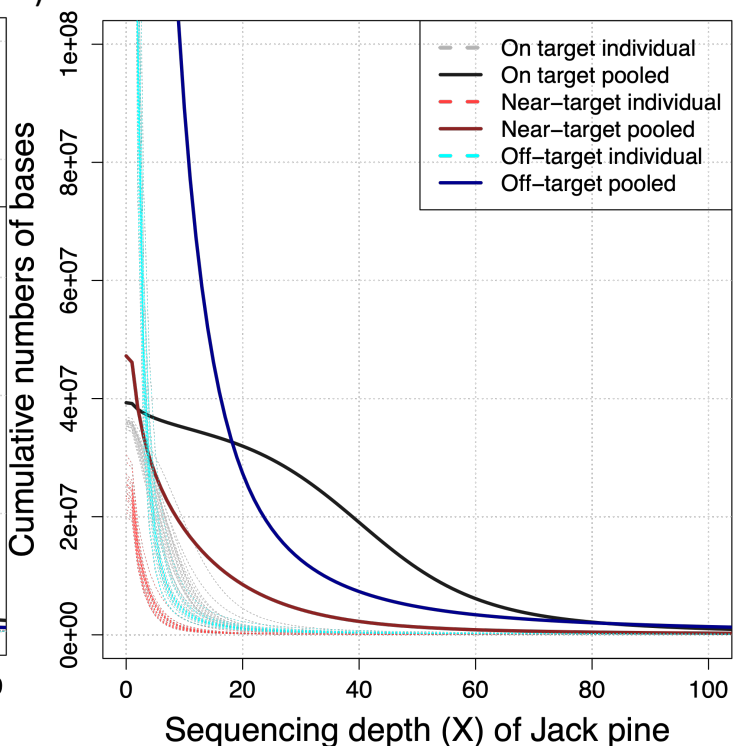

FIGURE 2 Cumulative numbers of bases in Douglas-fir (A) and jack pine (B) on target, near-target ( $\leqslant 500$ bp from target), and off-target regions. Dashed lines represent the cumulative numbers of bases in each of 20 indSeq samples. Solid lines represent the cumulative numbers of bases in the poolseq sample.

292 over 40 million bases in target regions with at least 20X sequencing depth (Figure 2A).

293 For jack pine poolSeq, we obtained over 30 million bases in on-target regions with at least

294 20X sequencing depth (Figure 2B). Sequencing depths in near- and off-target regions were

295 dramatically diminished compared to the on-target regions.

The total number of SNPs after baseline filtering varied across datasets and species

298 (Table 2). Douglas-fir generally had a higher number of SNPs called than jack pine, except

299 for poolSeq data. However, there were more jack pine megaSNPs intersecting with poolSeq

300 (25,500 SNPs) and indSeq (7,408 SNPs) than for Douglas-fir datasets (respectively 825

301 SNPs and 293 SNPs). Given that megaSNPs are cases where a heterozygote call was made 
TABLE 2 Output of SNPs from the conifer datasets. The intersection across all three baseline-filtered datasets were 7,006 SNPs for jack pine (JP) and 248 SNPs for Douglas-fir (DF).

\begin{tabular}{l|c|c|c|c}
\hline Dataset & Species & Baseline-filtered SNPs & \multicolumn{2}{|c}{ Baseline Intersecting SNPs } \\
& & & poolSeq & megaSeq $^{\dagger}$ \\
\hline indSeq & DF & $1,526,554$ & 636,279 & 293 \\
& JP & 377,080 & 255,706 & 7,408 \\
\hline poolSeq & DF & $1,601,285$ & - & - \\
& JP & $3,686,528$ & & - \\
megaSeq $^{\dagger}$ & DF & 398,774 & 825 & - \\
& JP & 32,751 & 25,500 & \\
\hline
\end{tabular}

† These numbers reflect only heterozygous SNPs (i.e., megaSNPs)

302 from a haploid sample and are therefore indicators of bioinformatic-paralogy errors, this

303 suggests that the error rate is much higher in jack pine. In total, several hundred thousand

304 SNPs were found in the intersection of poolSeq and indSeq for Douglas-fir (636,279 SNPs)

305 and jack pine (255,706 SNPs; Table 2).

\section{3 / Validation of megaSNPS as indicators of paralogy artifacts}

Upon inspection of our intersecting sets, patterns expected for duplicated and

311 genes. For instance, SNPs in duplicated genes should be most distinct from SNPs in

312 singletons when the derived allele is at intermediate frequency, and diverged duplicates

313 most distinct from singletons when the derived allele is fixed (Figure 3A). Sites consistent

314 with expectations for singleton, duplicates, and diverged duplicates were apparent from

315 intersection of poolSeq and indSeq sites (i.e., intersection I2; Figure 3D-E), while the 
bioRxiv preprint doi: https://doi.org/10.1101/2020.10.07.329961; this version posted October 8, 2020. The copyright holder for this preprint (which was not certified by peer review) is the author/funder. All rights reserved. No reuse allowed without permission.

Lind et al.

Validating pool-seq data in conifers
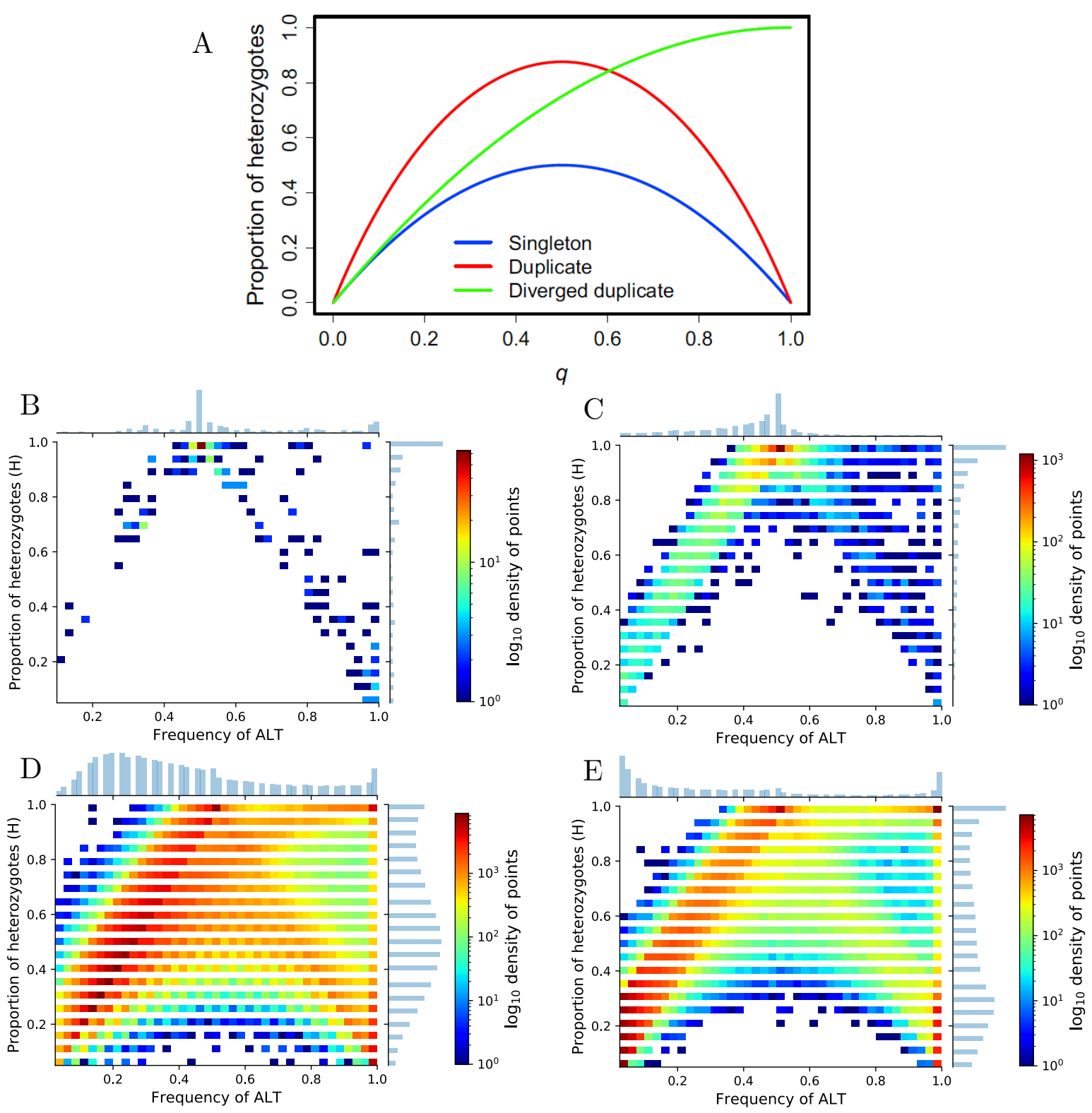

FIGURE 3 The proportion of heterozygotes, $H$, and the alternative (ALT) frequency calculated from indSeq data distinguish paralog misalignments according to expectations (A, Fig. 1 from McKinney et al. 2017), and empirically for Douglas-fir (B, D) and jack pine (C, E). B-C) Empirical distribution of megaSNP sites (candidate paralog sites identified as heterozygote calls from haploid tissue) calculated using indSeq data for those sites that were also called in poolSeq data (i.e., intersection I1). D-E) Empirical distribution of intersection I2 (indSeq and poolSeq intersection) calculated using indSeq data. Note color scale changes for each figure to accentuate patterns in the data. Frequency of ALT was binned for visualization purposes. Code to create these figures is available online: https://github.com/CoAdapTree/testdata_validation

316 indSeq sites intersecting with candidate paralog sites (megaSNPs, i.e., intersection I1) 
Lind et al.

Validating pool-seq data in conifers

317 displayed elevated levels of heterozygosity as expected from paralogs (Figure 3B-C).

318 Indeed, patterns of deviated allele ratios were also seen in our data (Figures S1 D-E, S2

319 D-E), where the vast majority of megaSNP sites were considerably different than the 1:1

320 read ratio expected of heterozygous diploids (Figures S1B-C, S2B-C) as would otherwise

321 be expected for singletons (Figures S1A, S2A). Lastly, when considering the standardized

322 allele ratio deviation ( $z$-score) we recover the same patterns of point clouds classified by

323 McKinney et al. (2017). We observe a dense set of SNPs around the $z$-score of 0.0 for $H$

324 values of 0.0-0.6 (Figure 4D-E) expected from singleton sites (Figure 4A), another set of

325 SNPs with elevated $H$ and/or $z$-score (Figure 4B-C) that is expected from duplicate loci

326 (Figure 4A), and a third set of SNPs with $H>0.9$ (Figure 4B-C) that is expected for

327 diverged duplicates (compare to Figures 5 and 8 in McKinney et al. 2017).

328 3.4 / Comparisons of sequencing approaches Loci within the intersection of baseline-filtered indSeq and poolSeq datasets (i.e.,

330 intersection I2) showed a strong positive association between allele frequencies estimated

331 from indSeq and poolSeq (Pearson's $r=0.9760, p=0.0000$ for jack pine; Pearson's $r=$

$3320.9483, p=0.0000$ for Douglas-fir; Figure 5A-B). Comparison of the MAF spectrum from

333 these estimates also revealed good agreement by frequency bins (Supplemental Figure S3).

334 After exploring various filtering strategies (see Supplemental Note 1.4), we applied filters 
bioRxiv preprint doi: https://doi.org/10.1101/2020.10.07.329961; this version posted October 8, 2020. The copyright holder for this preprint (which was not certified by peer review) is the author/funder. All rights reserved. No reuse allowed without permission.

Lind et al.

Validating pool-seq data in conifers
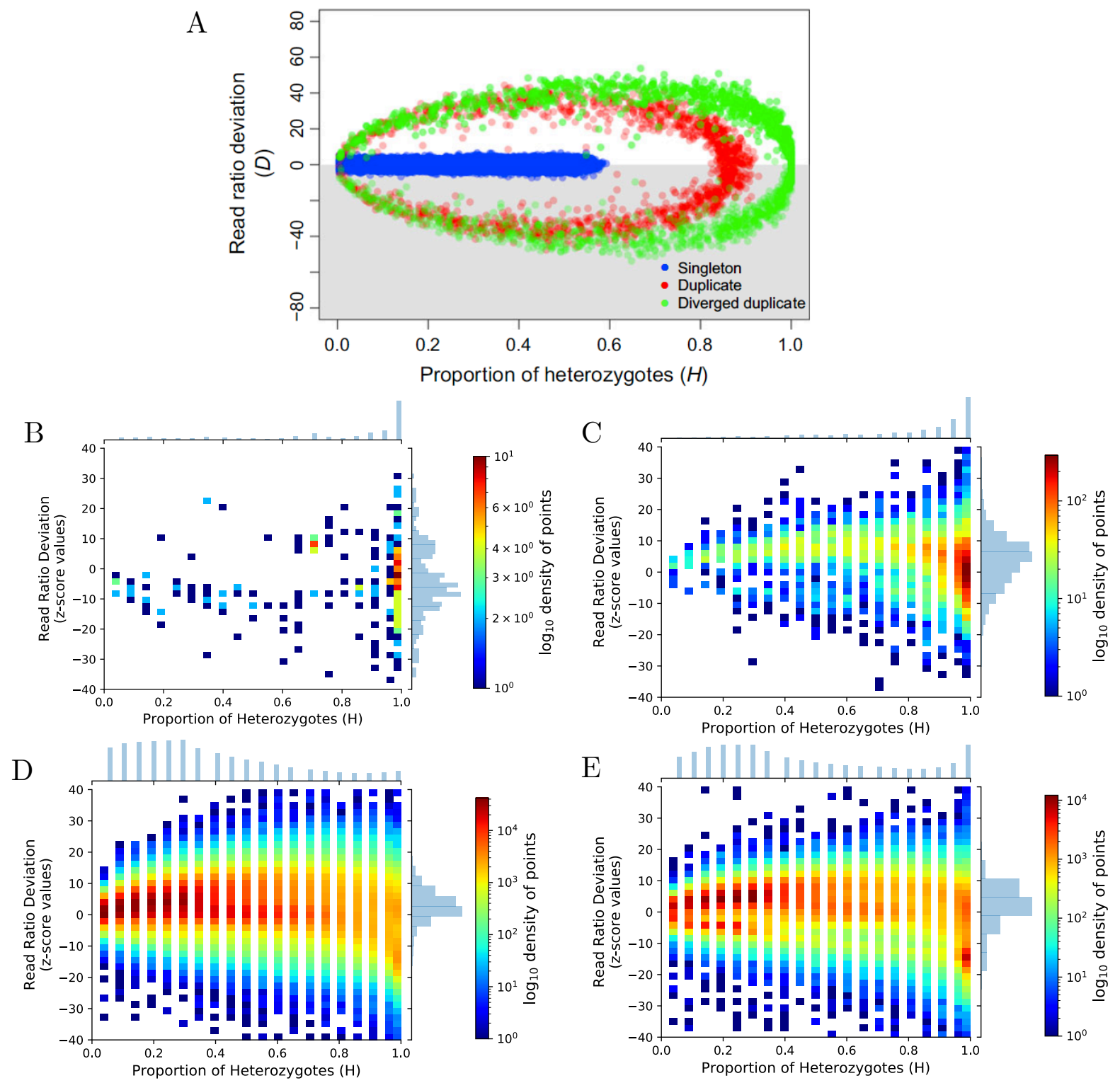

FIGURE 4 Standard deviation of read ratio $(z$-score) and the percentage of heterozygotes $(H)$ calculated from indSeq data distinguish paralog misalignments according to expectations (A, Fig. 4 from McKinney et al. 2017), and empirically for Douglas-fir (B, D) and jack pine (C, E). B-C) Empirical distribution of megaSNP sites (candidate paralog sites identified as heterozygote calls from haploid tissue) calculated using indSeq data for those sites that were also called in poolSeq data (i.e, intersection I1). D-E) Empirical distribution of intersection I2 (indSeq and poolSeq intersection) calculated using indSeq data. As with $3 \mathrm{~A}$, the distribution found in the gray box in $4 \mathrm{~A}$ will be found in the upper white panel because we used the reference allele instead of randomly choosing the allele. Note color scale changes for each figure to accentuate patterns in the data. Code to create these figures is available online: https://github.com/CoAdapTree/testdata_validation

335 that showed a positive effect on congruence between allele frequency estimates in our data 
Lind et al.

Validating pool-seq data in conifers

336 (removing megaSNP sites and indSeq sites with $H>0.6$ ); that resulted in removing sites

337 with extreme values of $A F d i f f$ (removing indSeq sites with $z$-score $>10$; filtering $H>$

$338 \quad 0.6$ alone also had this effect); and, finally, that gave us the best estimate of indSeq allele

339 frequency - our standard of comparison - and thus the best impression of the performance

340 of our poolSeq approach (removing indSeq sites with $>20 \%$ missing data). The correlation

341 of allele frequencies estimated from indSeq and poolSeq data increased after this filtering

342 (Pearson's $r=0.9907, p=0.0000$ for jack pine; Pearson's $r=0.9732, p=0.0000$ for

343 Douglas-fir) with relatively fewer sites with extreme differences in the estimates from each

344 method (see top-left and bottom-right corners of 2D histograms; Figure 5A-D). While

345 some differences remain in the estimates of the minor allele frequency spectrum (Figure

346 5E-F), these two methods largely agree, suggesting a robust poolSeq dataset for further

347 biological hypothesis testing.

\section{$348 \quad 4$ | Discussion}

$349 \quad$ Validation of pool-seq approaches commonly involves model organisms with complete

350 or near-complete chromosome-scale reference genomes (e.g., see Table 1 in Rellstab et al.

351 2013). However, there are few studies that explore this congruence in non-model organisms

352 such as conifers with large and highly fragmented reference genomes, and histories of

353 whole genome duplications and gene family evolution. Here we show that combining

354 exome capture and pool-seq can be an efficient method for quantifying genetic 
bioRxiv preprint doi: https://doi.org/10.1101/2020.10.07.329961; this version posted October 8, 2020. The copyright holder for this preprint (which was not certified by peer review) is the author/funder. All rights reserved. No reuse allowed without permission.

Lind et al.

Validating pool-seq data in conifers

A

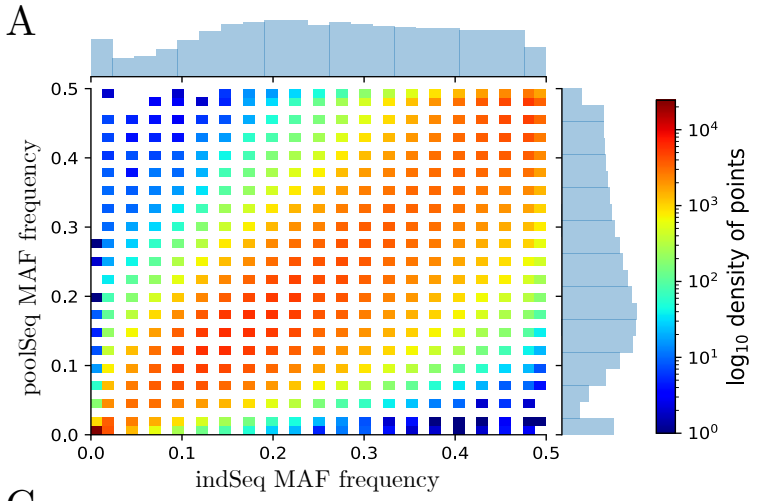

$\mathrm{C}$

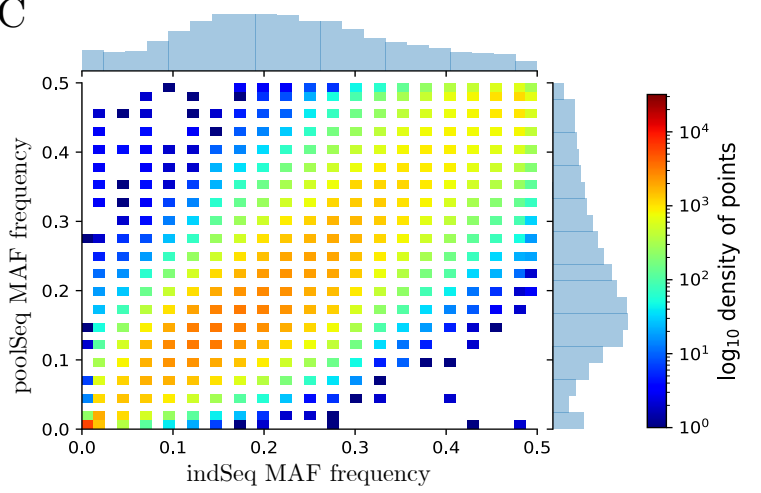

B

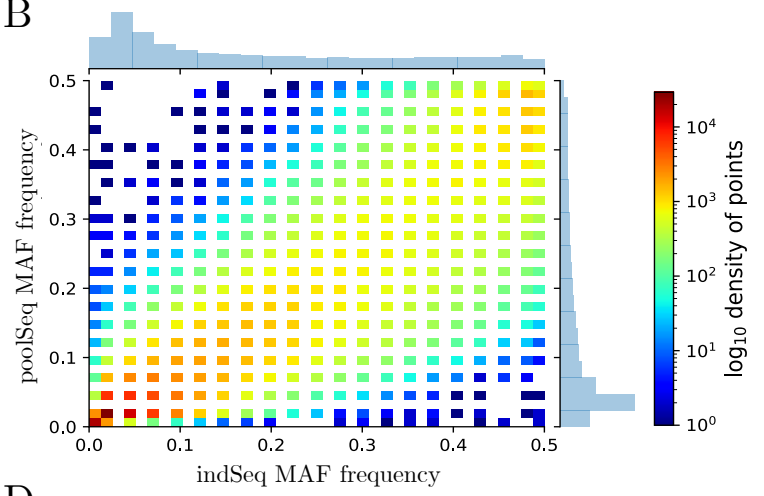

$\mathrm{D}$

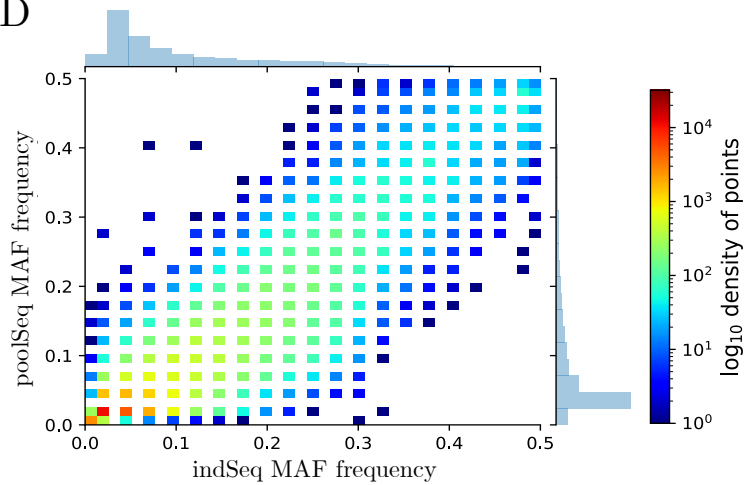

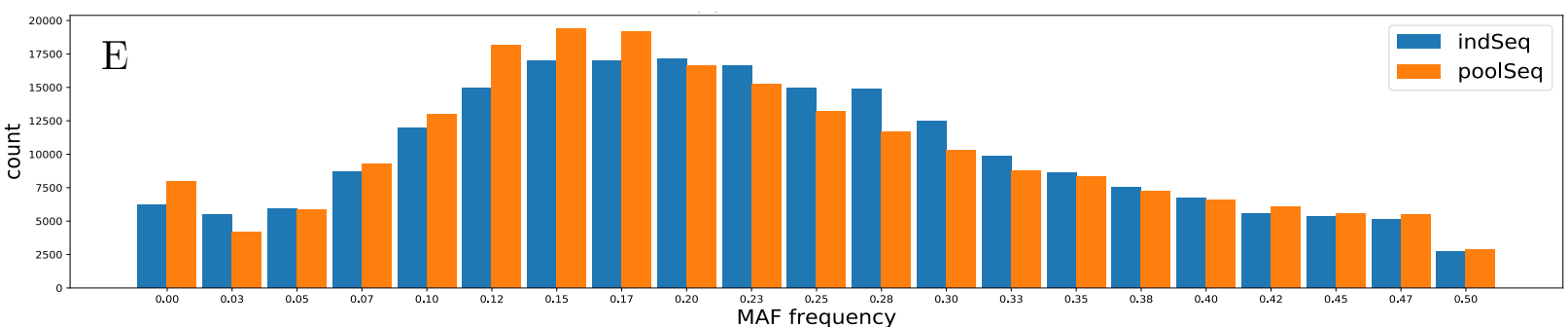

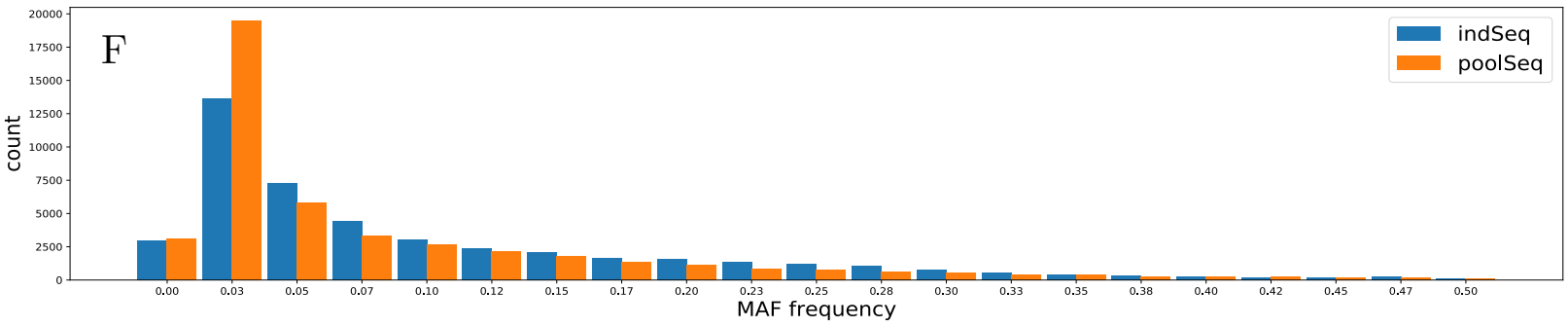

FIGURE 5 Congruence between indSeq and poolSeq (respectively $\mathrm{x}$ - and y-axes, A-D) minor allele frequency (MAF) estimates from Douglas-fir (A, C, E) and jack pine (B, D, F). A-B) 2D histogram of baseline-filtered intersection between indSeq and poolSeq (i.e., intersection I2). C-D) 2D histogram for SNPs after filtering intersection I2 for megaSNP sites, $H>0.6, a b s(z$-score) $>10$, and indSeq sites with $>20 \%$ missing data. E-F) Congruence of minor allele frequency spectra from SNPs in (C) and (D). Color scale is standardized to visualize differences in density between filtering steps. Minor allele frequency was binned for visualization purposes. Code to create these figures is available online: https://github.com/CoAdapTree/testdata_validation

355 polymorphisms in two such species, and that heterozygous SNPs from haploid data 
Lind et al.

Validating pool-seq data in conifers

356 (megaSNPs) consistently uncover sites with patterns expected from the misalignment of

357 paralogs (Figures 3-4, S1-S2). Further, we appear to uncover more false-positive variation

358 in jack pine than in Douglas-fir (Table 2). Concordance of allele frequency estimates from

359 baseline-filtered indSeq and poolSeq datasets (i.e., intersection I2) was strong $(r>0.948)$,

360 and increased with increased filtering, including the filtering of potential false-positive

361 sites $(r>0.973$, Figure 5$)$. These values are well within the range expected from previous

362 pool-seq studies (Table 1 in Rellstab et al. 2013), and in some cases perform better than

363 these model organisms.

364 Despite their role in adaptation and speciation (Lynch \& Conery 2000; Allendorf et al.

365 2015), the exclusion of potentially paralogous sites from next generation sequencing

366 datasets is commonplace due to the difficulty in differentiating genetic polymorphisms

367 from differences present among copies from single or diverged gene families (Dou et al.

368 2012; Hohenlohe et al. 2012; Dufresne et al. 2014). There are several methods by which

369 to detect such problematic sites, such as filtering by coverage (Dou et al. 2012), disomic

370 models such as Hardy-Weinberg proportions (Hohenlohe et al. 2011; Catchen et al. 2013;

371 Chen et al. 2014), or gene annotation, though there are several shortcomings (see

372 descriptions of these shortcomings in Table 1 of McKinney et al. 2017). When individual

373 sequencing data is available for the same individuals or populations, such information can

374 be used to isolate potentially paralogous sites from pool-seq exome capture studies (e.g.,

375 Rellstab et al. 2019). However, a potentially cost-saving alternative would be to sequence 
Lind et al.

Validating pool-seq data in conifers

376 the haploid tissue of a single individual (if available). Even so, there may be reduced

377 power to detect recently diverged paralogs (i.e., when derived alleles are at low frequency

378 and therefore not readily detected in a single individual). As such, heterozygous SNPs

379 called from our haploid data (megaSNPs) allowed us to identify variation from paralogous

380 misalignments that infrequently displayed patterns expected of singleton gene copies.

381 Indeed, high quality heterozygous calls from haploid sequencing are a reliable method for

382 identifying misalignments due the known monoallelic state of the sequenced site (Limborg

383 et al. 2016). While metrics from sequences of individuals are reliable (McKinney et al.

384 2017), they can falsely flag potentially paralogous sites as SNPs due to the stochastic

385 nature of the sequencing process and may result in the exclusion of biologically meaningful

386 information.

387 As pointed out by Rellstab et al. (2019), use of exome capture in many Pinaceae 388 species will require particular care to exclude potentially paralogous sites from

389 downstream analysis to avoid biased results. This is particularly true for pool-seq datasets

390 relying on read counts for allele frequency estimation or population genetic inferences such

391 as genotype-environment association (e.g., as implemented in baypass, Gautier et al.

392 2015). While individually sequenced datasets may be one path forward to identifying such

393 problematic sites (as in Rellstab et al. 2019, Shu \& Moran 2020), it will be cost-prohibitive

394 for large projects with many populations. As such, the sequencing of sufficient quantities

395 of DNA from haploid gametophyte tissue available for some plants, including conifers, 
396 seedless vascular plants, and bryophytes, offers an alternate path forward to balance

397 sequencing cost and data reliability.

\section{Acknowledgments}

399 The CoAdapTree project is funded by Genome Canada (241REF), Genome BC and 16 other

400 sponsors (http://coadaptree.forestry.ubc.ca/sponsors/), including Genome Alberta, Génome

401 Québec, the BC Ministry of Forests, Natural Resources Operations and Rural Development, and

402 Compute Canada. We thank Centre d'expertise et de services Génome Québec for sequencing

403 service, University of Calgary Information Technologies for system support, and Dr. Pia Smets

404 and Christine Chourmouzis for technical assistance. We also thank CoAdapTree Scientific

405 Advisory Board members John Davis, Matias Kirst, and Graham Coop for their guidance. Dr.

406 Sam Yeaman is also funded by the Natural Sciences and Engineering Research Council of Canada

407 (NSERC) and Alberta Innovates. Dr. Sally Aitken is funded by an NSERC Discovery Grant. The

408 funding bodies did not have any role in the design of the study, collection, analysis, or 409 interpretation of data in writing the manuscript. 
Lind et al.

Validating pool-seq data in conifers

\section{References}

$411 \quad A$.

412 Aitken SN, S Yeaman, JA Holliday, T Wang, S Curtis-McLane (2008) Adaptation, 413 migration or extirpation: climate change outcomes for tree populations. Evolutionary $414 \quad$ Applications 1:95-111.

Alberto FJ, SN Aitken, R Alía (2013) Potential for evolutionary responses to climate change-evidence from tree populations. Global Change Biology 19:1645-1661.

Allendorf FW, S Bassham, WA Cresko, MT Limborg, LW Seeb. JE Seeb (2015) Effects of crossovers between homeologs on inheritance and population genomics in polyploid-derived salmonid fishes. Journal of Heredity 106:217-227.

C.

Casola C, TE Koralewski (2018) Pinaceae show elevated rates of gene turnover that are robust to incomplete gene annotation. Plant Journal 95:862-876.

Catchen J, PA Hohenlohe, S Bassham, A Amores, WA Cresko (2013) Stacks: an analysis tool set for population genomics. Molecular Ecology 22:3124-3140.

Chen N, CV Van Hout, S Gottipati, AG Clark (2014) Using Mendelian inheritance to improve high-throughput SNP discovery. Genetics 198:847-857.

Chen S, Y Zhou, Y Chen, J Gu (2018) fastp: an ultra-fast all-in-one FASTQ preprocessor. Bioinformatics 34: i884-i890.

[dataset] Cronn R, PC Dolan, S Jogdeo, JL Wegrzyn, DB Neale, JB Clair, DR Denver (2017a).3B24_fc1136_ln7_GTGGCC. Sequence Read Archive of the National Center for Biotechnology Information (NCBI SRA). psme_diurnal3B-2400. BioSample: SAMN04168923; Sample name: diurnal3B-2400; SRA: SRS1117152.

[dataset] Cronn R, PC Dolan, S Jogdeo, JL Wegrzyn, DB Neale, JB Clair, DR Denver (2017b). Multi-genotype \#2 (MG2_I). Sequence Read Archive of the National Center for Biotechnology Information (NCBI SRA). Multi-genotype \#2. BioSample: SAMN00849794; Sample name: MG2; SRA: SRS308266. 
Lind et al.

Validating pool-seq data in conifers

$D$.

445 Danecek P, A Auton, G Abecasis, CA Albers, E Banks, MA DePristo, R Handsaker, G Lunter, G Marth, ST Sherry, G McVean, R Durban, 1000 Genomes Project Analysis Group (2011) The Variant Call Format and VCFtools. Bioinformatics 27:2156-2158.

Dou J, X Zhao, X Fu, W Jiao, N Wang, L Zhang, X Hu, S Wang, Z Bao (2012)

Dufresne F, M Stift, R Vergilino, BK Mable (2014) Recent progress and challenges in population genetics of polyploid organisms: an overview of current state-of-the-art molecular and statistical tools. Molecular Ecology 23:40-69.

$F$.

Fracassetti, M, PC Griffin, Y Willi (2015) Validation of pooled whole-genome resequencing in Arabidopsis lyrata. PLOS ONE 10:e0140462-15.

Futschik A, C Schlötterer (2010) The next generation of molecular markers from massively parallel sequencing of pooled DNA samples. Genetics 186:207-218.

$G$.

Gauter M (2015) Genome-wide scan for adaptive divergence and association with population-specific covariates. Genetics 201:1555-1579.

Gautier M, J Foucaud, K Gharbi, T Cézard, M Galan, A Loiseau, M Thomson, P Pudlo, C Kerdelhué, A Estoup (2013) Estimation of population allele frequencies from next-generation sequencing data: pool-versus individual-based genotyping. Molecular Ecology 22:3766-3779.

$H$.

Grabherr MG, BJ Haas, M Yassour, JZ Levin, DA Thompson, I Amit, X Adiconis, L Fan, R Raychowdhury, Q Zeng, Z Chen, E Mauceli, N Hacohen, A Gnirke, N Rhind, F di Palma, BW Birren, C Nusbaum, K Lindblad-Toh, N Friedman, A Regev (2011) Trinity: reconstructing a full-length transcriptome without a genome from RNA-Seq data. Nature Biotechnology 29:644-652. Pseudotsuga menziesii; RNA-Seq". Sequence Read Archive of the National Center for 
Lind et al.

Validating pool-seq data in conifers

Biotechnology Information (NCBI SRA). LA9S7. BioSample: SAMN04111182; SRA: SRS1089467; GEO: GSM1893337.

Hohenlohe PA, SJ Amish, JM Catchen, FW Allendorf, G Luikart (2011) Nextgeneration RAD sequencing identifies thousands of SNPs for assessing hybridization between rainbow and westslope cutthroat trout. Molecular Ecology Resources 11:117-122.

Hohenlohe PA, J Catchen, WA Cresko (2012) Population genomic analysis of model and non-model organisms using sequenced RAD tags. Methods in Molecular Biology 888:235-260.

Holliday JA, SN Aitken, JE Cooke, B Fady, SC González-Martínez, M Heuertz, JP Jaramillo-Correa, C Lexer, M Staton, RW Whetten, C Plomion (2017) Advances in ecological genomics in forest trees and applications to genetic resources conservation and breeding. Molecular Ecology. 26:706-17.

\section{$J$.}

Janes JK, JA Hamilton (2017) Mixing it up: the role of hybridization in forest management and conservation under climate change. Forests 8:237.

\section{$K$.}

Koboldt D, Q Zhang, D LarsoN, D Shen, M McLellan, L Lin, C Miller, R Mardis, L Ding, R Wilson (2012) VarScan 2: Somatic mutation and copy number alteration discovery in cancer by exome sequencing Genome Research 22:568-576.

Kovach A, JL Wegrzyn, G Parra, C Holt, GE Bruening, CA Loopstra, J Hartigan, M Yandell, CH Langley, I Korf, DB Neale (2010) The Pinus taeda genome is characterized by diverse and highly diverged repetitive sequences. BMC Genomics 11:420.

Krutovsky, KV, M Troggio, GR Brown, KD Jermstad, DB Neale (2004) Comparative mapping in the Pinaceae. Genetics 168:447-461.

\section{$L$.}

Li B, CN Dewey (2011) RSEM: accurate transcript quantification from RNA-Seq data with or without a reference genome. BMC Bioinformatics 12:323. 
Lind et al.

Validating pool-seq data in conifers

Li H, Durbin R (2009) Fast and accurate short read alignment with Burrows-Wheeler Transform. Bioinformatics 25:1754-60.

Li H, B Handsaker, A Wysoker, T Fennell, J Ruan, N Homer, G Marth, G Abecasis, R Durbin, 1000 Genome Project Data Processing Subgroup (2009) The sequence alignment/map format and SAMtools. Bioinformatics 25:2078-2079.

Li Z, AE Baniaga, EB Sessa, M Scascitelli, SW Graham, LH Rieseberg, MS Barker (2015) Early genome duplications in conifers and other seed plants. Science Advances 1:e1501084.

Lind BM, M Menon, CE Bolte, TM Faske, AJ Eckert (2018) The genomics of local adaptation in trees: Are we out of the woods yet? Tree Genetics \& Genomes 14: 29.

Limborg MT, LW Seeb, JE Seeb (2016) Sorting duplicated loci disentangles complexities of polyploid genomes masked by genotyping by sequencing. Molecular Ecology 25:2117-2129.

[dataset] Little SA, IG Boyes, K Donaleshen, P von Aderkas, J Ehlting (2016) RNA-Seq of Douglas-fir megagametophytes at different development points and of cone bracts and scales. Sequence Read Archive of the National Center for Biotechnology Information (NCBI SRA). June10_Poll. BioSample: SAMN05255397; SRA: SRS1697751; GEO: GSM2202773.

Liu Y-Y, K-Z Yang, X-X Wei, X-Q Wang (2016) Revisiting the phosphatidylethanolamine-binding protein (PEBP) gene family reveals cryptic FLOWERING LOCUS T gene homologs in gymnosperms and sheds new light on functional evolution. New Phytologist 212:730-744.

Lu M, KV Krutovsky, CD Nelson, TE Koralewski, TD Byram, CA Loopstra (2016) Exome genotyping, linkage disequilibrium and population structure in loblolly pine (Pinus taeda L.). BMC genomics. 17:1-1.

Lynch M, JS Conery (2000) The evolutionary fate and consequences of duplicated genes. Science 290:1151-1155.

$M$.

McKenna A, M Hanna, E Banks, A Sivachenko, K Cibulskis, A Kernytsky, K Garimella, D Altshuler, S Gabriel, M Daly, MA DePristo (2010) The Genome 
Lind et al.

Validating pool-seq data in conifers

Analysis Toolkit: a MapReduce framework for analyzing next-generation DNA sequencing data. Genome Research 20:1297-1303.

[dataset] McKinney GJ, RK Waples, LW Seeb. JE Seeb. (2016) Data from: Paralogs are revealed by proportion of heterozygotes and deviations in read ratios in genotyping by sequencing data from natural populations. Dryad Data Repository, https://doi.org/10.5061/dryad.cm08

McKinney GJ, RK Waples, LW Seeb, JE Seeb (2017) Paralogs are revealed by proportion of heterozygotes and deviations in read ratios in genotyping-bysequencing data from natural populations. Molecular Ecology Resources 17:656-669.

Morse AM, DG Peterson, MN Islam-Faridi, KE Smith, Z Magbanua, SA Garcia, TL Kubisiak, HV Amerson, JE Carlson, CD Nelson, JM Davis (2009) Evolution of genome size and complexity in Pinus. PLOS ONE 4:e4332.

$N$.

Neale DB, A Kremer (2011) Forest tree genomics: growing resources and applications. Nature Reviews Genetics 12:111-122.

Neale DB, PJ Martínez-García, AR De La Torre, S Montanari, X-X Wei (2017a) Novel Insights into Tree Biology and Genome Evolution as Revealed Through Genomics. Annual Review of Plant Biology 68:457-483.

Neale DB, PE McGuire, NC Wheeler, KA Stevens, MW Crepeau, C Cardeno, AV Zimin, D Puiu, GM Pertea, UU Sezen, C Casola, TE Koralewski, R Paul, D Gonzalez-Ibeas, S Zaman, R Cronn, M Yandell, C Holt, CH Langley, JA Yorke, SL Salzberg, JL Wegrzyn (2017b) The Douglas-fir genome sequence reveals specialization of the photosynthetic apparatus in Pinaceae. G3: GENES, GENOMES, GENETICS 7:3157-3167.

Neale DB, O Savolainen (2004) Association genetics of complex traits in conifers. Trends Plant Sci 9:325-330.

\section{$P$.}

Parchman TL, JP Jahner, KA Uckele, LM Galland, AJ Eckert (2018) RADseq approaches and applications for forest tree genetics. Tree Genetics \& Genomes 14:39. 
Lind et al.

Validating pool-seq data in conifers

589 Pavy N, M Lamothe, B Pelgas, F Gagnon, I Birol, J Bohlmann, J Mackay, N Isabel, J

Puritz JB, KE Lotterhos (2018) Expressed exome capture sequencing: A method for cost - effective exome sequencing for all organisms. Molecular Ecology Resources 18:1209-1222.

$Q$.

Quinlan AR, IM Hall (2010) BEDTools: a flexible suite of utilities for comparing

\section{$R$.}

R Core Team. 2018. R: A language and environment for statistical computing. R genomic features. Bioinformatics 26:841-842.

$S$. sequencing and pooled exome capture to study local adaptation in the giga-genome of Pinus cembra. Molecular Ecology Resources 19:536-551.

Rellstab C, S Zoller, A Tedder, F Gugerli, MC Fischer (2013) Validation of SNP allele frequencies determined by pooled next-generation sequencing in natural populations of a non-model plant species. PLOS ONE 8:e80422.

Shu M, EV Moran (2020) Testing pipelines for genome-wide SNP calling from Genotyping-By-Sequencing (GBS) data for Pinus ponderosa. Researchsquare. https://doi.org/10.21203/rs.3.rs-32336/v1

Simão FA, RM Waterhouse, P Ioannidis, EV Kriventseva, EM Zdobnov (2015) BUSCO: assessing genome assembly and annotation completeness with single-copy orthologs. Bioinformatics 31:3210-3212.

Schlötterer C, R Tobler, R Kofler, V Nolte (2014) Sequencing pools of individualsmining genome-wide polymorphism data without big funding. Nature Reviews Genetics. 15:749-63.

Sniezko RA, LA Winn (2017) Conservation and restoration of forest trees impacted by non-native pathogens: the role of genetics and tree improvement. InIn: Sniezko, 
Lind et al.

Validating pool-seq data in conifers

625

Richard A.; Man, Gary; Hipkins, Valerie; Woeste, Keith; Gwaze, David; Kliejunas, John T.; McTeague, Brianna A., tech. cords. 2017. Gene conservation of tree species - banking on the future. Proceedings of a workshop. Gen. Tech. Rep. PNWGTR-963. Portland, OR: US Department of Agriculture, Forest Service, Pacific Northwest Research Station. p. 68. 2017 (Vol. 963).

Suren, H, KA Hodgins, S Yeaman, KA Nurkowski, P Smets, LH Rieseberg, SN Aitken, and JA Holliday (2016) Exome capture from the spruce and pine giga-genomes. Molecular Ecology Resources 16: 1136-1146.

$V$.

Voronova A, B Viktorija, A Korica, D Rungis (2017) Retrotransposon distribution and copy number variation in gymnosperm genomes. Tree Genetics \& Genomes 13:88.

\section{$W$.}

Wegrzyn JL, JD Liechty, KA Stevens, LS Wu, CA Loopstra, HA Vasquez-Gross, WM Dougherty, BY Lin, JJ Zieve, PJ Martínez-García, C Holt (2014) Unique features of the loblolly pine (Pinus taeda L.) megagenome revealed through sequence annotation. Genetics 196:891-909.

Wu TD, Watanabe CK (2005) GMAP: a genomic mapping and alignment program for mRNA and EST sequences. Bioinformatics 21:1859-1875.

$Y$

Yeaman S, KA Hodgins, KE Lotterhos, H Suren, S Nadeau, JC Degner, KA Nurkowski, P Smets, T Wang, LK Gray, KJ Liepe (2016) Convergent local adaptation to climate in distantly related conifers. Science. 353:1431-3. 
Lind et al.

Validating pool-seq data in conifers

\section{Data Accessibility}

650 At the time of submission, all code needed to process raw sequence data through figure

651 generation has been made available on GitHub ${ }^{1,2}$. Commands used in probe design used

652 default arguments unless otherwise specified. Upon acceptance, a 'release' of all code

653 included with submission will be created and remain on GitHub, and will be subsequently

654 archived on DataDryad.org.

655 Genomic and RNA sequence data will be deposited on the Sequence Read Archive of the

656 National Center for Biotechnology Information (NCBI SRA) as well as the Gene Expression

657 Omnibus. Nimblegen SeqCap probes are available for jack pine under design name

658 180215_jackpine_v1_EZ_HX1, and for Douglas-fir under design name

659 80215_DOUGFIR_V1_EZ.

\section{Author Contributions}

661 SA and SY obtained funding for the study. SA, SY, BL, and ML conceived the research

662 design with contributions from TB. DOV generated the sequence data which BL analyzed,

663 with contributions from ML, PS, and TB. ML designed probes with contributions from SY.

664 BL and ML designed SNP calling pipelines, which were coded by BL and reviewed by ML.

665 BL wrote the manuscript with contributions from ML. All authors contributed to the

666 editing of this manuscript.

\footnotetext{
1 https://github.com/CoAdapTree/varscan_pipeline

2 https://github.com/CoAdapTree/testdata_validation
} 